\title{
Identificador: Viaje a Perú
}

Título: Forma en que se puede disponer el viaje del Perú, toca también a algo de gobierno y hase de ver algunas veces

Lugar: Perú

Fecha: Entre 1617 y 1682

Tipo: Relación, recomendaciones

Editor: Lucía Riopedre Ferreira, Marta Fernández Alcaide

Descripción: Relación de bienes que deben llevarse para realizar el viaje al Perú y motivos por los que se mencionan. Se incluyen consejos sobre el gobierno.

DOI: https://dx.doi.org/10.12795/Viaje_a_Peru

$\{$ h 1r $\}\{1\}$ Por la grandeza deV<uestra $>$ ex<celencia $>$ autoridad del cargo es ffuerza se haga laxornada de aqui $\{2\}$ a la enbarcacion con toda autoridad. y despues de enbarcado en la mar conlapusi $\{3\}$ ble. y al desenbarcar en cartagena portouelo y panama. toda la que se pudiere y $\{4\}$ en la otra mar. Toda la que quisiere pues ua por sujurisdición y enbajeles $\{5\}$ propios y ellos y el general y capitanes a su orden y dispusición y prouision

\{6\} El usaje de la persona. a deser litera cochey mula dere $\{7\}$ galo para el dia o rrato que hiziere bueno y sereno.

$\{8\}$ Los criados nonbrados y pajes. a mula. dando acada yen $\{9\}$ tilonbre unaparaun criado y otra para cada criado de $\{10\}$ seruicio y una para todos los lacayos.

$\{11\}$ dos azemilas para la cama.

$\{12\}$ [margen: las caxas de la plata con sus apartadijos] dos para la plata, y dinero del gasto, del camino

$\{13\}$ quatro para la rrecamara del uso del camino

$\{14\}$ una para fiambreras y cantimploras con nieue

$\{15\}$ otra para hachas. Por si la jornada. Llegare a la noche

$\{16\}$ otrapara la reposteria y mesa. y bujias para el camino

$\{17\}$ otrapara sillas y bufetes.

$\{18\}$ otrapara lacozina. 
$\{19\}$ otraparablandones en sus cajas. si los oui<ese> y si no alla se haran

$\{20\}$ La demas Recamara y de criados en carros D azemilas $\{21\}$ las que fueran menester. Conforme lo queouiere.

$\{22\}$ [subrayado: y todos los baules. o caxas. no a depesar ninguna mas $\{23\}$ de quatro arrobas. porque no podran yr de portouelo a $\{24\}$ Panama y por ningun caso enbie $\mathrm{v}<$ uestra $>$ ex $<$ celencia $>$ RopaPor $\{25\}$ el rrio de chagre que ademas delpeligro tienen despues $\{26\}$ cinco leguas de cruzes apanama muy trauajosas de $\{27\}$ de cuestas y lodos errios, y cuesto dineros y no es a horo]

$\{28\}$ [margen, subrayado: Tanbien a de lleuar v<uestra $>$ ex $<$ celencia $>$ arnes de $\mid \mathrm{a}$ cauallo y deynfante bueno y luzido] Para las espadas. se hara caxon largo a sutamaño que $\{29\}$ no pese mas ded<ichas $>$ quatro arobas,

$\{30\}$ esta casa caminara [subrayado: toda lleuando un alguazil] que haga $\{31\}$ el aposento y aqui. y hecho pasara a tenerlo aparejado $\{32\}$ [margen: Puedeyr el ueedor tanbien o unjen|tilonbre.] y aloxado. todo. en la siguiente xornada. y desta ma\{33\}nera estarinaproueydas las posadas deaqui aseuilla $\{34\}$ y puede yr un ayudante de cozina con el alguazil parate $\{35\}$ ner. aperejada la cenay comida delotro dia. y seescu $\{36\}$ chara hazer dos casas. quesuele azerse por mejor

$\{37\}$ en Seuilla se podra preuenir con carta deaqui la casadel $\{38\}$ duquede medina q<ue>stauazia [subrayado: o la alcacar que esta a dis $\{39\}$ pusicion del condedeoliuares.] o buscar casa particular

$\{\mathrm{h} 1 \mathrm{v}\}\{1\}$ de seuilla se adeyr a san lucar Porel Rio en la faluca $\{2\}$ de la casa dela contratacion quetiene tendal y sinoestu\{3\} uiere alli hazer que latraygan para eltal dia. o sino en $\{4\}$ tres barcos luengos con rremeros y toldo. quees me $\{5\}$ jor que portierra $y$ demenos gasto tres partes y sea $\{6\}$ delleuar de seuilla la comida Parav<uestra> ex<celencia> y Criados.

$\{7\}$ en san lucar aposentara el duque av<uestra $>$ ex<celencia $>$ que lo $\operatorname{acos}\{8\}$ tumbray [margen: esto a de estar preuenido antes de $\mid$ yr y el duque enbiara jentilonbre | a v<uestra> ex<celencia> [subrayado: y si no lo hiziere o al cami|no. yr derecho a santodomingo]] sino conbidare consucassa. en santodomingo $\{9\}$ estara $\mathrm{v}<$ uestra> ex<celencia> bienospedado deaposento y los criados $\{10\}$ en posadas $\mathrm{o}$ mesones que serapoco $\mathrm{t}<$ iempo $>$ eldealli. 
\{11\} y si la enbarcaçion ffuere a cadis vayase aenbarcar $\{12\} \quad v<$ uestra> ex<celencia> alpuerto desanta maria y atrauiese labaya $\{13\}$ en barco delos delaues y no enotra enbarcaçion oen $\{14\}$ un coche con quatro criados porlapuente desuaco y $\{15\}$ seyra a aposentar [subrayado: a san ffrancisco] si el $g<$ ouernador> de alli no hiziere

\{16\} $\mathrm{V}<$ uestra> ex<celencia> se a de enbarcar en la capita delos galeones adond $<\mathrm{e}>\{17\}$ [margen: el general a de yr. en Capitana tan|bien. pero aunque todo vaaorden $\mid$ del uirrey, le dejarav<uestra $>$ ex < celencia $>$ el gouierno] adeyr porseñor deella en la camara depopa escojien $<\mathrm{do}>\{18\}$ si lleuara dos corredores el de abajo asiporque es may<or> $\{19\}$ como por quetendra menos balançe. esta adeyzada $\{20\}$ reçada con algunos. Taffetanes o damasquillos de la $\mathrm{g}$ [doblez] $\{21\}$ na si ya noouiera enlaguardarropa alguna colgadu<ra $>\{22\}$ vieja de que poder hazer alguna de dos barasymedi $<a>\{23\}$ delargo $<$ po $>$ co mas. y veyntey ocho o treynta deruedo que $\{24\}$ Paraesto qualquierabastara. yesfuerça uaya de aderecada

\{25\} Los criados que sean de enbarcar conv<uestra $>$ ex $<$ celencia> en la ca $\{26\}$ pitana [margen: noes bien vayan mas criados | por sucomodidad. ni son menester] an de ser los fforçosos elmayordomo y ueedor $\{27\}$ el camarero y un gentilonbre decamara y un moço de camara. quatro pajes. mastresalay copero. cozi $\{28\}$ nero y moço. Repostero deplata y estrado. botiller y $\{29\}$ dos lacayos quesiruan enlo que se dirá y coneste $\{30\}$ acompañamiento de criados yrav<uestra> ex<celencia> yellos bien $\{31\}$ seruido y acomodados. y los demas criados yran en $\{32\}$ los galeones quel general les señalare.

\{33\} Paraeladorno y buenolor de la camara ay que escuse av<uestra alteza> $\{34\}$ dealmadiamiento quesera pusible lo este. porel mal olor $\{35\}$ del nauio [subrayado: sea delleuar juncia con una plecao baçia $\{36\}$ de plata grande muy bien adereçada y un binco que $\{$ h $2 \mathrm{r}\}\{1\}$ siempre [tachado: siempre] se a de traer. en la mano con agua de $\{2\}$ Rosay uinagre Rosado.] [margen: ya podia ser no almadiarse $\mathrm{v}<$ uestra $>$ ex $<$ celencia $>$ | pero si lo hiziere no se escuse el bo|mito que es saludable con el caldo | o alguna sustancia] $\mathrm{y}$ siestono bastare y todauiase $\{3\}$ almadiare $\mathrm{v}<$ uestra $>$ ex $<$ celencia $>$ setenga cuydado deponer a cozer $\{4\}$ un aue contocino garuancos ajos y muchaesPeçia $\{5\}$ y yr tomando amenudo tragos delcaldo echandole $\{6\}$ dentro unayema deun gueuo cadaues y aunquese $\{7\}$ buelua boluer atomarotros. aunquesea Porfuerca $\{8\}$ y no lepese av<uestra> ex $<$ celencia $>$ del bomito queconellos quedarasano $\{9\}$ y en este $t<$ iempo $>$ no seade usar 
deotro Remedio aunquelo $\{10\}$ diga galeno porque no nauego y esto es esPeriençia $\{11\}$ y assi lo hize conel conde de Lemos mi señor y mi s<eñora $>$.

\{12\} [subrayado: Para la cama se haran un par dehazericos o cabecales $\{13\}$ detaffetan llenos derrosay otros. Perfumes yolor $\{14\}$ de manera que conellos. este la cabeça confortada]

$\{15\}$ Para la cama se haran enseuilla dos sauanas deba $\{16\}$ dana pegados sin costura queyalos saben hazer y $\{17\}$ echar uno debajo de la sauanay otro encima delaotra $\{18\}$ es limpio ffresco y seescusan chinches y no estan bu[***] \{19\} cuero turco paraesto para uestido esbueno.

$\{20\}$ [subrayado: Lacamisa seade mudarcadadia]. porelcalor y porelescru $\{21\}$ pulo depiojos quesecrian muchos y yaq<ue $>\mathrm{v}<$ uestra $>$ ex $<$ celencia $>$ no $1<$ os $>\{22\}$ crie se lepodran pegar aunqueno no saliendo dela cama<ra>

$\{23\}$ el uestido para la mar sea ligero y eljubon estafado $\{24\}$ solo elestomago y estose escusara sino hiziere dañ $<0>\{25\}$ pueden ser dexergueta perpetuam o algunade $[* * *]\{26\}$ ordinaria por que es la calor muy grande en la $[* * *]$

$\{27\}$ el corredor vaya siempre cubierto con un tend $[* * *]\{28\}$ cana $[* * *]$ por amor deel sol que daporalli.

\{29\} [margen: esto se entiende cuando el medico | ffuere Rogado y nouasallo] Siv<uestra> ex<celencia> lleuare medico abradeyr enbarcado $c[* * *]\{30\}$ personasy le dara su mesa quel virey de napoles $[* * *]\{31\}$ se lo daria por siacaso ffuere necesario que no sea

\{32\} [subrayado: Podria ser quel general coma conv<uestra> ex<celencia> alo menos $\{33\}$ ffuerça se leapriete aquelo haga] no entendiendo es $\{34\}$ cumplimiento sino mucho gusto y porquelleuanca $\{35\}$ maradas podra suplir sulugar elcapitan deynfanter $<\mathrm{ia}>$

$\{\mathrm{h} \mathrm{2v}\}\{1\}$ Para la mesay boca dev<uestra $>$ ex $<$ celencia $>$ se an de meter 300 galli $\{2\}$ nas. entres gallineros y ocho hanigas de [tachado: mais] trigo para su $\{3\}$ comida y tendra quenta conellas un lacayo Paradarles $\{4\}$ de comer y beber y rrecojer los gueuos quepusieren fres $\{5\} \cos$ que es en la mar muy gran Regalo y por mayor El $\{6\}$ mayordomoLoadeui sitar cada dia que es la comidaprinçipal. 
$\{7\}$ Treyntaperniles de rruti o algarrobillas porque no sean $\{8\}$ muy salados y se esteraran de en dos en dos. cuero con c<uero $>\{9\}$ Porqueno se apolillen o crien gusanos y uayan conserua

$\{10\}$ Una dozena de Carneros muy buenos y unpar deter $\{11\}$ neras [margen: bien pudieray destomas pero Como | seles muda el pasto. senfflaquecen | y no suele auer lugar para mas porq<ue> | lleua el general para su mesay ca|maradas. pero pueden yr enotronauio] y conestos tendra quenta eLotro lacayo de dar $\{12\}$ les decomer Cada diay de beber delamacamora del gale $\{13\}$ on. y eLagua Laadedartanbien El galeon

$\{14\}$ dos çebones muy buenos Los quales sean de adereçar to $\{15\}$ do Lo magro de ellos en sallchichasy longanizas muy $\{16\}$ bienadereçadas y echallas cada cosa deporsi en orças de $\{17\}$ Barro bedriado y deretir todolo gordo y enpellas de los $\{18\}$ çebones y he $<$ n $>$ chir con ella las orças. y conesto yran $\operatorname{con}\{19\}$ seruadas todo el camino. y es buenrregalo en la mar

$\{20\}$ dos toçinos enteros para adereçar de comer. mechan $\{21\}$ a uer cozer las ollas. y lo demas que se aderecare.

$\{22\} 50$ pares deperdizes ffrescas muy bienperdigadas y $\{23\}$ [margen: la mejor comida es esta si se sabe a|derecar. y de mayor Regalo.] Echaldas asimismo enorças y llenalles todo el gueco $\{24\}$ con manteca siLa ouiere deuacay sino depuerco con $\{25\}$ algun gusto de sal. y dara cada diavna asada av<uestra $>m<$ erced $>\{26\}$ ques muy grande Regalo.

$\{27\}$ [margen: $[* * *$ ]nque deste genero vayamas no $\mid[* *$ ] porta quesepuede hazer plato deello] dos dozenas de lenguas de uacasaLadas para Los $\{28\}$ sabados y cinquenta depuerco en adobo. y ahu $\{29\}$ madas quesean muy buenas y es deregalo.

$\{30\}$ media dozena de cuñetas deatundeyzada. buenos $\{31\}$ y un barril deatundebadana para los criados.

\{32\} [margen: no es esta como laqueaqui seuende / pero es comida muy sana] cantidad de mielgas. quese hallaran enseuilla $\{33\}$ que es comidaregaladay muy sana.

$\{34\}$ un quintal deuacallao ffresco lo mas que fuere $\{35\}$ pusible y medio de pescada cicial para los Criados. que $\{36\}$ van con $v<$ uestra $>$ ex $<$ celencia $>y$ tal ues haranplato dello para laboca 
$\{\mathrm{h} 3 \mathrm{r}\}\{1\}$ Arros, garuanço. Lanteja. ffideos. son muy buenos $\{2\}$ en La mar, de cada cosa vna hanega que en la otra mar sirue

\{3\} [margen: Siruen pare el uiernes guisadas | con dulçe. o paratostadas.] dos dozenas de caLabaças grandes curadas que llaman $\{4\}$ deLas yndias. que entreti[tachado: ni] enen por uerdura porq<ue> $\{5\}$ en La mar no se puede[tachado: $n]$ conseruar y ajos y cebollas

$\{6\}$ vn aroba de miel blanca.

$\{7\}$ vn quintal se pasa dealmuneca en un barriL o sera

$\{8\}$ vn quintal dealmendra. moriscapara Principio $\{9\}$ manjar blanco y adereçar de comer y si sobra no sepierde.

$\{10\}$ vn quintal de açucar blanco enpilon

$\{11\}$ dos arobas de orejones. de durasno quatro que no se pierde

$\{12\}$ [margen, subrayado: el hinojo es bueno en la mar yela|nis. para de noche y de dia al beber] vn quintal de anis mostazay hinojo confitado pa\{13\}ra acabar de comer beber entre dia y para desayunarse.

$\{14\}$ cinquenta $q<$ uintales> de guindas en almibar. en 50 olleras $\{15\}$ de uidro deaLibra. es muy cordial conserua en lamar

$\{16\}$ [margen, subrayado: Conserua desta no se hallara en carta|xena ni en panamay mucha no sepierde] Caxas de menbrillo perada durasno y cidra y bocadillos $\{17\}$ de lo mismo. y las caxas Pequenas y muchas.

$\{18\}$ alcaparras. ynperiales y alcaparones chiquitos enuina $\{19\}$ gre. $q<u e>$ smuy apetitoso en Lamar mucho que no lo ay alla

$\{20\}$ Tallos de alcaparra en uinagreparaensaLadaes buena

$\{21\}$ [margen, subrayado: deste genero se lleue Lo que se a de comer y| a la mesa para sus años.] azeytunas gordales de aLcaLa uerdes y moradas y $\{22\}$ otras de mançanilla azofairadas todo entero.

$\{23\}$ cien arobas de azeite Lunecas dozientas para que $\{24\}$ sealpiru / el quesobrare paraLa mesa de $\mathrm{v}<$ uestra $>$ ex $<$ celencia $>$. 
$\{25\}$ [margen: deste genero de uino para los criados | setomara secacalla.] 50 botijas de uino de monterque a Laneso guada $\{26\}$ canal. para que se sirua en La mesa alos guespedes $\operatorname{si}\{27\}$ v<uestra> ex<celencia> no Lo bebe. aunque sera neçesario un trago en $1[* * *]$

$\{28\}$ quatro botijas de uino tinto de jere $\mathrm{q}<\mathrm{ue}>$ srregalada $[* * *]\{29\}$ Para una sopa aLguna ues y seuaria el gusto.

$\{30\} 300$ gueuos en sal. para adereçar de comer y L[***] \{31\}nes. con los quales y los de las gallinas abra basta $<$ nte $>[* * *]$

\{32\} [margen: naranjas no duran tanto [subrayado: como | los limones pero es mejor agrio.] 500 Limones poncies. con sus Palillos. en aren[***] $\{33\}$ llegaran frescos. y algunas naranjas. entre el[ $[* *]\{34\}$ y algunas dulçes desan Lucar debarameda

$\{35\}$ si se hallaren melones deynnierno en seuilla o en $\{36\}$ coria se tomaran para principio. $\mathrm{q}<\mathrm{ue}>\mathrm{son}$ frescos los $\mathrm{q}<\mathrm{ue}>\mathrm{ou}[* * *]$

\{37\} Peros y camuesas para Los primeros dias que no duraran $\{38\}$ mucho en lamar pero cualquieracosa deste genero sabebien

$\{\mathrm{h} 3 \mathrm{v}\}\{1\}$ manteca de uacas. si se hallare es muy buenacon acu $\{2\}$ car. y bien lauada paraprincipio

$\{3\}$ q<ue $>$ so de ffLandes. o deotro que sea de Lo muy bueno alguno $\{4\}$ que es buen postre, y buen matalotaje y para guisar de comer

\{5\} [subrayado: Para que coma $\mathrm{v}<$ uestra> ex<celencia> pan fresco cada dia o de dos en $\{6\}$ dos días que estodo Regalo en Lamar. sean de lleuar $\{7\}$ quatro fanegas de harina en barriles medio quinta $\{8\}$ lenos. bien çernida y muy apretadapor que nose pi $\{9\}$ erday en barriles estancos. eL cozinero sera como a $\{10\}$ de ser el horno de cobre y mientras mas chico mejor]

\{11\} dozequintaLes debiscocho blanco candial que siso $\{12\}$ brare seruirapara La otramar y quiça sabrabien a $v<$ uestra $>$ a $<$ lteza $>$

\{13\} Tanbien semeteran ocho odiez quintaLes depan $\{14\}$ [margen, subrayado: deste genero mucho es bueno que $\mid$ si sobra sirue en laotramar que no $\mid$ se halla y silo ay muy caro] terciado Paraquecoma el serui<cio $>$ ordinario $q<\mathrm{ue}>\mathrm{va}$ con $\mathrm{v}<$ uestra $>$ $\mathrm{a}<$ lteza $>$ 
\{15\} Para Los demas criados se les metera algunos car $\{16\}$ neros. cien aues.quintales de biscocho Los diez $\{17\}$ blanco y Lo demas. terciado [subrayado: q<ue $>$ Lo quesobrare seruira $\{18\}$ Para laotramar.]

\{19\} Perniles y tocino de araçena. [subrayado: carne salada si laay $\{20\}$ deyrlanda.] q<ue>s mejor que deseuilla. vacallao. cicial $\{21\}$ aro haua garuanco lantexa queso Pasa e higo $\{22\}$ azeyte y uino y vinagre. algunas conseruas. para $\{23\}$ los enffermos. un moco decozina que les aderece $\{24\}$ de comer y otros dos Lacayos. que los siruan. y sus $\{25\}$ criados que lleuaren. y seacomodaran o todos jun $\{26\}$ tos o haran camaradas. arranchor. como lespareçiere $\{27\}$ dos uinetes de atun deyxada dos bariles de debada $\{28\}$ na. dos quintales deuacallao azeytunas yalca $\{29\}$ Parras. q<ue>s muy buen matalotaje ajoyçebolla mucho [tachado: y uino y bingre]

$\{30\}$ en Las yslas de barlouento no se desenbarque $\mathrm{v}<$ uestra $>$ ex $<$ celencia $>\{31\}$ que suele auer algunos yndios de guera enboscados y $\{32\}$ no es seguro el Lugar. alli refrescaran el agua $\mathrm{q}<\mathrm{ue}>\mathrm{s}$ buena

$\{33\}$ [subrayado: y en san lucar meteran Parav<uestra> ex<celencia> treyntabonjas de ba\{34\}ro por bedriar ni enpegar llenas del agua deLalgarobo o de $\{35\}$ La quebebe eL duque que muy buena y asi noolera]

$\{36\}$ [subrayado: Para beber frio aunque dizen no es muy sano se lleuaran $\{37\}$ dos quintales de salitrey garafas y cubite demadera pa\{38\}ra enffriar o cantinplora de plata muy delgada]

$\{\mathrm{h} 4 \mathrm{r}\}\{1\}$ [margen: el de las Casas $\mathrm{R}<$ eales>, es el mas fresco $\mid \mathrm{y}$ de los conuentos [subrayado: san francisco]] en cartaxenaportobelo y panama daran a $\mathrm{v}<$ uestra $>$ ex<celencia $>$ elaloja $\{2\}$ miento mejor que ouiere y sera fresco no siendo asia $\{3\}$ gusto [subrayado: en los conuentos. abra buenaloxamiento.]

\{4\} [margen: el salir a hazerexercicio sera muy | de manana o sobre tarde. aduierto | quel sereno deprima noche noes sano] Procure $v<$ uestra $>$ ex $<$ celencia $>$ noledeel sol pues no tiene aque salir y $\{5\}$ siempre. seuestira detodo punto antes que salga de su ca $\{6\}$ maray entodoeL dia no se desnude decomo uistiere la mañana

$\{7\}$ en Leuantandose $v<$ uestra> ex<celencia>comaunbocado dealgo aunque noeste $\{8\}$ en uso deello no es saludable estar enayunas y pro $\{9\}$ cure $v<$ uestra excelencia>. 
quanto pudiere no çenar denoche o silo hizi $\{10\}$ ere no sea cosa decarne. y siensalada cozidagueuos frescos $\{11\}$ o pescado ffresco, es lo mas saludable enestas tierras calientes.

$\{12\}$ Si algun matalotaje sobrare delo q<ue>s regalos yuiscocho y $\{13\}$ harinay conseruas seguardara para laotramarque $\{14\}$ no se hallara a conprar en aquellatierra

$\{15\}$ ase delleuar dos o tres arobas de açafran para todo elt $<$ iempo $>$ que $\{16\}$ alla estuuierev<uestra $>$ ex $<$ celencia $>$ que no Lo ay en elpiru.

\{17\} Parael camino canela clauo pimienta nuez despecie $\{18\}$ ael parecer del cozinero quesabe elgusto de $v<$ uestra $>$ ex $<$ celencia $>$ y azaffran

\{19\} Elagua sea siempre cozida q<ue>smalsana y enestos lugares $\{20\}$ Pocos xarros de agua entre dia ni a secas sin tomar algun $\{21\}$ dulçe para beber. que no es sano.y es mejor enuinada.

$\{22\}$ El uestido de lamar sera de xergueta peretuam u otra cosilla $\{23\}$ aestetono o de seda lijera.

$\{24\}$ [margen, subrayado: esto sea de entender yndistinta $\mid$ mente talues. o tal no. pero si fulere. arma salga $v<$ uestra> ex<celencia> y pidalesa|quenlas armas. que dara gesto a la | ynffanteria. que loydo murmu|rar de lo contrario y uistelo.] un capote de canpana para los aguaceros no porq $<\mathrm{ue}>\mathrm{v}<\mathrm{uestra}>\mathrm{ex}<$ celencia $>\{25\}$ adeestar ael pero porconseje deun criado. si ouiere alguno $\{26\}$ [subrayado: sea $v<$ uestra> ex<celencia> elprimero que saliere a la cubierta en qual $\{27\}$ quier Refriega o ocasion de arma. que se gana fama y $\{28\}$ nombre y entrejente Ruin no conuiene dejen denotaresto $\{29\}$ q<ue $>$ se celebrara eternamente y por el contrario lo demas]

\{30\} [margen, subrayado: Pero laprimera bocalmente lo | mande $\mathrm{v}<\mathrm{uestra}>$ ex<celencia> quelooygan ellos] [subrayado: auiendo algun aguacero les mandara darv<uestra> ex<celencia> a los $\{31\}$ marineros y soldados acada una botija deuino ordina $\{32\}$ rio y algun uiscocho blanco basta quel mayordomo lo diga $\{33\}$ y hagay esteaduertido desto deunaues para siempre]

$\{34\}$ [margen, subrayado: una dozena desonbreros. lleue $v<$ uestra> ex <celencia> | hechos en seuilla quepesen a quatro $\mid$ oncas. cadauno y auisarcon $\mathrm{t}<$ iempo $>\mid$ hagan que sonparalima buenos.] enestastierras el uestido ligero y en lima ligero y graue $\{35\}$ como sotanilla alarodilla hereruelo sin enbargo debajo $\{36\}$ sepuedetraer color telao bordado. 
\{37\} El uiaje depanama alima sera aun mas agusto aunque $\{38\}$ [margen, subrayado: en estaoCasion no a decomer nadie | conv<uestra> ex<celencia> asistirantodos a lamesa] no entan grandes bajeles. sonpropios y a orden de $\mathrm{v}<$ uestra> ex<celencia> $\{39\}$ todos. los ministros y de su nonbramiento para $\mathrm{v}<$ uestra> ex<celencia> ga $\{40\} 1$ linas terneras y no coma $\mathrm{v}<$ uestra $>$ ex $<$ celencia $>$ carnero por ningun caso. $\{41\}$ Pero delo quealli ouiere se ordenara como lo desta mar

$\{\mathrm{h} 4 \mathrm{v}\}\{1\}$ Al cozinero sele mandara enseui<lla $>$ conpre a su gusto todo $\{2\}$ el cobre queouiere menester parauna grandiosa cozina

\{3\} [subrayado: La bajilla $\mathrm{q}<\mathrm{ue}>\mathrm{v}<$ uestra> ex<celencia> lleuare seabastante paraocho odres $\{4\}$ de mesa no ser agusto sepidaprestada queel dinero que en $\{5\}$ esto segasta en casasequeda y es autoridad no pedir enlcam<ino>

$\{6\}$ [margen, subrayado: esto se entiende enlima porq<ue> | allino ay mocos de Cauallos sino | negros y yndios no ualennada | y layerua no a de costar dinero] El cauallerizo. tendra a su cargo el buscar elrepar $\{7\}$ timiento de lalfalfa yerua que comen los cauallos $\{8\}$ siemPre y pidira dos o quatro yndios derepartimiento $\{9\}$ Para Laregar y q<ue>la acarreen y [tachado: sigu] lleben a lacaualleriza $\{10\}$ en mulas o rozines que ade auer para ello. y asimismo $\{11\}$ de su guadarnes. y depedir los negros neçesarios confor $\{12\}$ me la caualleriza. quisiere tener $v<$ uestra $>$ ex $<$ celencia $>$.

\{13\} [subrayado: al callao no adeyr $\mathrm{v}<$ uestra $>$ ex $<$ celencia $>$ en $\mathrm{t}<$ iempo $>$ deocasion en coche sino $\{14\}$ en un muy buen cauallo yotro de dieztro por la grande $\{15\}$ za y con [mag<estad> pero con la mayor afabilidad del mundo por $\{16\}$ que le seguiratodo el [tachado: mundo] conesto y en aquella tierra $\{17\}$ ynporta gane $v<$ uestra> ex $<$ celencia $>$ nonbre de humano y le amaran $\{18\}$ y huya dequepor rriguroso leteman $\mathrm{q}<\mathrm{ue}>$ ledejaran solo]

\{19\} [subrayado: a menester $\mathrm{v}<$ uestra> ex<celencia> en esta Corte un agente tal yense $\{20\}$ uilla un corespondiente Rico y deuerdad. asi para lo $\{21\}$ que se leremitiere como para lo q<ue>sele pidiere ynbie $\{22\}$ de aca. $q<u e>$ sera necesario, y para que tenga hecho este $\{23\}$ matalotaje en sus $t<$ iempos $>$. y cosechay no aguarde a $\{24\}$ hazello porfebrero quecostara mas y no sera tanbueno]

$\{25\}$ a Los pajes no sescusa darles tres libreas una de paño para $\{26\}$ [margen: suelen los uirreyes dar librea de | sus colores alaguardia. esta la abra $\mid$ de dar $v<$ uestra> 
ex<celencia> negra por ser buido y de | aca. se puedelleuarpano negro para | ella de segouia de 30 o $32 \mathrm{R}<$ eales> bara | estasuuetodo el $\mathrm{t}<$ iempo> ] el camino otra de xergueta o perpetuan para seuilla $\{27\}$ salucar Cartaxina portouelo y panama y unanegra $\{28\}$ de sedapara entrar en lima, adonde solo aellos yal $\{29\}$ mastresala seles da de comer porque atodos se les ayuda $\{30\}$ Por su camino queay en quey luego se busca.

\{31\} [subrayado: Ropa de mesa muchay rrica y manteles de a 20 quarte $\{32\}$ les [margen: [subrayado: Para las pascuas el Rey da | ayuda de costa bastante] y elrey|no o coregidores enbian] o de a 15 y algunas. quatro tablas de a [tachado: a V] [***] $\{33\}$ para el dia quelaudiencia e ynquisicion comiere con $v<$ uestra $>$ ex $<$ celencia $>\{34\}$ o por las pascuas quees forcoso o tal ues queguste $\mathrm{v}<$ uestra $>$ ex $<$ celencia $>$ della

$\{35\}$ [subrayado: cera blanca en pan para gastar alla y en bujias y hachas 100 $\mathrm{q}<$ uintales $>\mathrm{q}<\mathrm{ue}>\mathrm{se}\{36\}$ ra menester y se lleuara sin fletes]

\{37\} [subrayado: el sitial parala yglesiapuede ser negro o morado con oro o sin el]

\{38\} [subrayado: para enbarcar y desenbarcar $\mathrm{v}<$ uestra> ex <celencia> se adelleuar untapete y almo $\{39\}$ hadarica. y ase delleuar un clarin o tronpeta $\mathrm{q}<\mathrm{ue}>$ haga senas $\{40\}$ y de camino paratocar al albor y acaualgar]

$\{$ h $5 \mathrm{r}\}$ [signo: +$]\{1\}$ Atreuimiento pareçiera quando un hombre tan poco prudente y menos $\{2\}$ entendido y no con mas esperiencia queotro quisiese dar consejo aquien $\{3\}$ por el mucho suyo lo puede dar a los muy sobrado delo que a mi me falta $\{4\}$ pero quien Conociere que mipadre y abuelo parientes e yo somos y emos si $\{5\}$ do criados de $v<$ uestra $>$ ex $<$ celencia $>$ y de los suyos demas de cien anos a estaparte. Jus $\{6\}$ gara ser auiso derrotero dado con buenzelo mouido delamornatural $\{7\}$ que los hijos dalgo tienen y deuentener aquien los Cria, alimenta honrra $\{8\}$ y ffaborece y pues para camino tanlargo pormar y tierra y tan desusado $\{9\}$ para $v<$ uestra $>$ excelencia $>$ es bien menester suplico $\mathrm{R}<\mathrm{eciba}>$ el que offrece mi boluntad.

$\{10\}$ Conocida cosaes que xornada tan larga prolija y peli $\{11\}$ grosa nace de deseo de seruir a surrey. y dejar descansar $\{12\}$ suestado y si ffuereposible desenpenarlo o añadirle $\{13\}$ Paralo qual es menester. aplicar medios saludables

\footnotetext{
${ }^{1}$ El final de esta línea, debido a la mala conservación de la tinta y a que está tachado, no se puede descifrar.
} 
$\{14\}$ Los criados. sean pocos. Conocidos esperimentados y va $\{15\}$ sallos pues por lo menos. seruiran con amor natural. $\{16\}$ Louno, para que se aprouechen. y con surriqueza honrren $\{17\}$ supatria y lootro para quelos naturales vezinos de $\{18\}$ aquellos Reynos seconsuelen Viendo que no lleuael $\{19\}$ virrey quienles coma el pan queganaron sus pagados, $\{20\}$ Causa Vigente para ser $v<$ uestra $>$ ex $<$ celencia $>$ amado. que es lo quede $\{21\}$ ue buscar. el buen principe. Consejo dejenoffonte.

\{22\} Los fforcosos. eynescusables. son. capitandelaguardia. $\{23\}$ mayordomo cauallerizo camarero condos xentiles hon $\{24\}$ bres y tres pajes. unsecretario. contador. maestresala $\{25\}$ seis pajes. copero. veedor. y los officiales. y una doze $\{26\}$ na de negros para Cauallerizay seruicio de lacassa. y $\{27\}$ unpar de mugeres. para el cuydado de lacassa y dela $\{28\}$ Persona y rregalo deella. Un medico aunque alla $\{29\}$ los ay pero si se puede. el queconoce la conplexion es $\{30\}$ mejor. un letrado queaya sido jues paraacesores fuerça

\{31\} Paralautoridad. del principe. ynportaria lleuar $\operatorname{Re}\{32\}$ Partidos entre estos criados media dozena de abitos $\{33\}$ Pues Tantos ay y sedan cada dia.

$\{34\}$ el trato del agente delpiru y detodas las yndias a de $\{35\}$ ser affable manso begnino y amoroso. que con esto $\{36\}$ encubre un uirrey lo que quiere. y los manda a ça $\{37\}$ Patazos porquelesuiuen con amor y buena boluntad $\{38\}$ tanto en las cosas de la guerra como enlapaz. y aun $\{39\}$ queles quiten la capa lo tienen por bien, pero si alg<uno> $\{40\}$ se des sabriere omostrare conesto altiues desacato $\{\mathrm{h} 5 \mathrm{v}\}\{1\}$ o Cosa que huela a leuantar los ojos nolo a de castigar $\mathrm{v}<$ uestra $>$ ex $<$ celencia $>\{2\}$ pero mandar a un alcalde de corte. lo castigue conynperio hu $\{3\}$ millde manso pero con mucha seueridad. segundon $\mathrm{m}<$ artin $>$ enriq $<$ ues $>$

$\{4\}$ y Paramas agradar alos vezinos y benemeritos sea el pri $\{5\}$ mer pregon degouernaçion mandar quetodos los que tienen $\{6\}$ cedulas de su $\mathrm{m}<$ agestad> de encomienda las presenten ante su secre $\{7\}$ tario para uellas y graduallas. Cosa conquetodos sealentaran $\{8\}$ aseruir y amar. a $v<$ uestra $>$ ex $<$ celencia $>$ louno paraque no lecansen y ellos $\{9\}$ ganen amigos enlatierra.

$\{10\}$ Los martes y uiernes por las mañanas asista $v<$ uestra $>$ ex $<$ celencia $>$ a la audien $\{11\}$ cia publica conlos oydores. y los jueues auer las Residencias $\{12\}$ Por la mañana y no se uea ninguna sinque estev<uestra $>$ ex<celencia $>$ Presente $\{13\}$ Para hazerse capas de los delitos que cometen los corregidores y $\{14\}$ hazer memoria dello 
para quando pidan quelos bueluan aocupar $\{15\}$ y hallarse en los acuerdos lunes y jueues enlatarde.

$\{16\}$ las cosas de gouierno y guerra yndistintamentetocan a $\{17\} \quad v<$ uestra> ex<celencia $>$ y las dejusticia al audiencia perotalvez. conparecer $\{18\}$ delacesor bien ffundado podra $v<$ uestra $>$ ex $<$ celencia $>$ enmendar algun mal $\{19\}$ abuso o agrauio pasion odio parcialidad o modo de malproceder $\{20\}$ Pocas bezes pero justifficadas y de manera que cobren miedo $\{21\}$ y Respeto suyapor bien no lo hizieren.

$\{22\}$ no consentir. se hagan los pleytos ynmortales antes procuren $\{23\}$ el despacho particular mente el delpobre y pedir testimonio $\{24\}$ si sepudiese que no ay pleyto que despachar pidiolo don martín $\{25\}$ enrriques en nueuaespaña.

$\{26\}$ hazer al deCano del audiencia no consienta que el procurador $\{27\}$ y letrado depobres y presos huelguen y onrrar mucho a los $\{28\}$ diputados dela caridad que acuden a esto ante el uirrey yan $\{29\}$ te el audiencia dandoles banquillo para esto solo y no mas. $\{30\}$ no se les llama de bos quando tratan deste ministerio ni de $m<$ erced>

\{31\} quando alguno pide rremuneraçión de seruicios Presen $\{32\}$ ta sus papeles. ante el secrettario degouernacion y Respon $\{33\}$ dera $v<$ uestra $>$ ex $<$ celencia $>$ alaparte por su secretario o haziendole $\mathrm{m}<$ erced $>\{34\}$ o mandandole siruamas y boluerle los papeles alsecret<ario $>\{35\}$ degouernaçion que es el que adedespachar la cedula o titulo $\{36\}$ delam<erced $>$ que $v<$ uestra $>$ ex $<$ celencia $>$ le hiziere.

$\{$ h 6r $\}\{1\}$ Los officios denoministraciones los a de dar $v<$ uestra $>$ ex $<$ celencia $>$ $\{2\}$ a sus criados sin que en ellas entre ningun. hijo ninieto $\{3\}$ de conquistador ni benemerito. y los officios de justiz $<i a>\{4\}$ del rreyno Proueav $<$ uestra $>$ ex $<$ celencia $>$ primero aalgunos delos bene $\{5\}$ meritos y despues aalgunos criados. de manera que $\{6\}$ vayan quatro primero que uno decasa Pero dalle al $\{7\}$ criado si ffuere tal el mejor y conesto se acomodatodo

$\{8\}$ Mande $v<$ uestra> ex < celencia> que las caxas decomunidades tengan $\{9\}$ llaues y llaueros una el correjidor otra el cura. otrael $\{10\}$ cacique conorden no se llegue aldinero deellas. sinlicen $\{11\}$ cia de $v<$ uestra $>$ ex $<$ celencia $>$ Pena de priuacion deofficio y nose holgaran $\{12\}$ tanto los nueuamenteproueydos porel consejo. 
$\{13\}$ alos oydores y ministros derropa les adedar $v<$ uestra $>$ ex $<$ celencia $>$ silla y $\{14\}$ les a de llamar de $m<$ erced> pero si ffuere zaino no sentarse con $\{15\}$ el ni cubrirse y se despachara luego.

$\{16\}$ a los caualleros de abito seles. adellamar de $m<$ erced $>$ qual uez $\{17\}$ y a los caualleros que ay en la ciudad algunos muy buenos $\{18\}$ aunque no tienen abito. honrre $\mathrm{v}<$ uestra $>$ ex $<$ celencia $>$ como lepareciere $\{19\}$ aunque no tienen $\mathrm{m}<$ erced $>$. perono bos. a secas. el marques $\{20\}$ de salinas antes que ffuese uirrey lleuo cedulaparaque $\{21\}$ don martin enrriques lellamase $\mathrm{m}<\mathrm{erced}>\mathrm{y}$ diese sillay lecho $\{22\}$ diez o doze boçes antes que se ladiera ni dijera $\mathrm{m}<$ erced $>$.

$\{23\}$ conlaynquisicion y arcobispo tengav $<$ uestra $>$ ex $<$ celencia $>$ muy grande $\{24\}$ y buena correspondencia se escusan en cuentos que aun $\{25\}$ que el uirrey puede mas noes justo ayaquexa de $v<$ uestra $>$ ex $<$ celencia $>$

$\{26\}$ aningun çaçerdote consienta. $v<$ uestra $>$ ex $<$ celencia $>$ searrodille ni este $\{27\}$ descaperucado hablando. y paralos principios el paje $\{28\}$ decamara delportero se lo aduertira que el uirrey don fr<ancisco $>\{29\}$ detoledo perdio elpremio desus seruicios poresto.

$\{30\}$ quando $v<$ uestra $>$ ex<celencia $>$ quisiere sacardinero delacaxao hazeral $\{31\}$ guna cosa del seruicio de su $m<$ agestad> o despachar jues para algo $\{32\}$ que no aya ordenespresa de su $m<$ agestad> - no lo haga. sin primero \{33\} llamar y hazer acuerdo. coneloydor masantiguo oelque $\{34\} \mathrm{v}<$ uestra $>$ ex $<$ celencia $>$ quisiere y ffiscal yofficiales $\mathrm{R}<$ eales> y juntos Por $\{35\}$ ante el secretario de gouierno setratara y sino uieniere $\{36\}$ en ello la mayor parte cese pero si a $v<$ uestra $>$ ex $<$ celencia $>$ todauia ay[* **] $\{37\}$ ciere que conuiene mandelo porauto y aunque con $[* * *]\{38\}$ gan lo hagay deauiso al rrey estando en lasilla y pro $\{39\}$ cure seaprueue enel c<onsejo $>$ noaguarde a la Residencia

$\{\mathrm{h} \mathrm{6v}\}\{1\}$ A los Contadores dequentas les guardarav<uestra $>$ ex $<$ celencia $>$ lo mismo $\{2\}$ que ouiere guardado y dado monterey. y los demas uireyes $\{3\}$ lauisita del cerro depotosi toca av<uestra> ex<celencia> esta seadecome $\{4\}$ ter apersona del Rey no quelo entienda

\{5\} La corresponsion detodas las cartas tocaal seCret<ario> dev<uestra> ex $<$ celencia $>$ 
$\{6\}$ Pidav<uestra $>$ ex<celencia $>$ al consejo cedula parapoder lleuar cada amo al \{7\} Piru quatro mill ducados de azeiteyuino y uestuario Para $\{8\}$ sucassa. que al uirrey don martin enRiques se dio de $2 \mathrm{U}\{9\}$ y esta cedula seacomo dara $\mathrm{e}<\mathrm{n}>$ Seu $<$ illa $>$ conel corespondiente $\{10\}$ quealli a de auer forcossamente

\{11\} Para ser auisado del bueno o mal gouierno a los ojos de los $\{12\}$ suditos a de traer $\mathrm{v}<$ uestra $>$ ex $<$ celencia $>$. dos. confidentes personas. Princi $\{13\}$ peles. y de autoridad. que oygan sepan consecreto lo que $\{14\}$ en la rripublica se murmura o alaba dev<uestra> ex<celencia $>$ y que $\{15\}$ cada noche le auisen paraoydo por $v<$ uestra $>$ ex $<$ celencia $>$ esaminesilo $\{16\}$ quese murmura es o no con Razon y enmiende lo que $\{17\}$ tuuiere enmienday quedeaduertido para adelante $\{18\}$ hizolo don martin enrriq<ues> en mex $<$ ico $>$ y lima y en napo $\{19\}$ es el duque dealcala el uiejo y elconde de lemos mi señor

Para el conde mi s<eñor $>[$ firma $]$

Forma en que se puede | disponer el uiage del |Peru toca tanbien | a algo de gobierno | y ase deber algunas bezes 